6. Consolidated framework for implementation research. Center for Clinical Management Research website. https://cfirguide.org/. Published 2017. Accessed Janaury 21, 2021.
7. Framework for human health risk assessment to inform decision making. US Environmental Protection Agency website. https://www.epa.gov/risk/ framework-human-health-risk-assessment-inform-decision-making. Published 2014. Accessed January 21, 2021.

\title{
Resistors and constipators: Financial impact in an academic medical center, a mathematical model
}

\author{
Kelly A. Reagan $\mathrm{BS}^{1}$ (1), David M. Chan $\mathrm{PhD}^{2}$, Ginger Vanhoozer BSN, RN, CCHM ${ }^{3}$, Rachel J. Pryor RN, MPH ${ }^{4}$, \\ Michelle E. Doll MD, MPH ${ }^{3}$, Emily J. Godbout DO, MPH ${ }^{3}$, Michael P. Stevens MD, MPH, FACP, FIDSA, FSHEA ${ }^{5,6,7,8}$ and \\ Gonzalo M. Bearman MD, MPH, FACP, FSHEA, FIDSA $8,9,10$ \\ ${ }^{1}$ Department of Mathematics and Applied Mathematics and the Department of Statistical Sciences and Operations Research, Virginia Commonwealth University, \\ Richmond, Virginia, ${ }^{2}$ Department of Mathematics and Applied Mathematics, Virginia Commonwealth University, Richmond, Virginia, ${ }^{3}$ Infection Prevention \\ Program, Virginia Commonwealth University Health System, Richmond, Virginia, ${ }^{4}$ Department of Infection Prevention, Virginia Commonwealth University Health \\ System, Richmond, Virginia, ${ }^{5}$ Internal Medicine Department, Virginia Commonwealth University, Richmond, Virginia, ${ }^{6}$ Division of Infectious Diseases, Virginia \\ Commonwealth University, Richmond, Virginia, ${ }^{7}$ Antimicrobial Stewardship Program, Virginia Commonwealth University Health System, Richmond, Virginia, \\ ${ }^{8}$ Virginia Commonwealth University, Richmond, Virginia, ${ }^{9}$ Division of Infectious Diseases, Virginia Commonwealth University, Richmond Virginia and \\ ${ }^{10}$ Epidemiology and Infection Control Division, Virginia Commonwealth University Health System, Richmond, Virginia
}

To the Editor-Hospital-acquired infections (HAIs) impact many hospitalized patients, and they have a high mortality rate. HAIs cost the US healthcare system billions of dollars every year. Active resistors and organizational constipators are in leadership positions and resist change. They often block and delay the adoption of best practices, which save money and lives. ${ }^{1}$

A strategy to overcome active resistors is to present scientific evidence supporting new practices. The use of standardized central-line bundle kits (SCLBKs) is an infection prevention program that has proven to reduce central-line bloodstream infections (CLABSIs). ${ }^{2}$ Bathing patients with a $2 \%$ chlorhexidine gluconate (CHG) solution reduces annual CLABSIs and catheter-associated urinary tract infections (CAUTIs). ${ }^{3}$ We have shown that delays in implementing and increasing CHG compliance results in additional HAIs and costs. ${ }^{3}$ Here, we focus on the delay of implementing SCLBK and CHG bathing on CLABSI and CAUTI infections. We calculated the impact of active resistors and organizational constipators on these infections over 5 years, and we present a cost analysis.

\section{Methods}

\section{Model structure}

A discrete-time Markov chain model was implemented in MATLAB to simulate patients moving through different patient classes. We defined 4 classes: patients with a central line, patients with a Foley catheter, and patients with both, and patients with neither. Patients with central lines may acquire CLABSIs, and patients with Foley catheters may acquire CAUTIs. The distribution of patients depends on the class they were in previously. The next day's distribution was calculated using the following formula:

Author for correspondence: Kelly A. Reagan E-mail: reaganka2@vcu.edu

Cite this article: Reagan KA, et al. (2022). Resistors and constipators: Financial impact in an academic medical center, a mathematical model. Infection Control \& Hospital Epidemiology, 43: 533-535, https://doi.org/10.1017/ice.2021.34

$$
X(t+1)=B \cdot I \cdot P \cdot D \cdot X(t)
$$

where $B$ is a transition matrix that represents the probability of getting CHG bathed or obtaining a SCLBK, $I$ is a transition matrix that represents the probability of getting an infection, $P$ is a transition matrix that represents the probability of obtaining a catheter or central line, and $D$ is a transition matrix that represents the probability of being discharged. Patients with CLABSI or CAUTI may develop a secondary infection of the other type. Each day, if a patient does not acquire an infection or an intervention, the patient moves to the $i+1$ version of the same class.

The patient's average length of stay, $1 / \delta_{\mathrm{k}}$, differs for each class $k$. The daily probability of getting an intervention $p, \rho_{p}$, was calculated using the following formula:

$$
\rho_{p}=1-(1-K)^{\delta_{k}}
$$

where $K$ represents the percentage of hospitalized patients with intervention $p$.

The infection rate, $r$, was calculated based on a compliance rate of $60 \%$ for CHG bathing and by number of days since last intervention. Here, $\eta$ and $\kappa$ are the reduction of incidence of CAUTI and CLABSI due to CHG bathing and CLABSI due to SCLBK, respectively, and were pre- to postintervention incidence.

\section{Model inputs}

Virginia Commonwealth University Health System is an 865-bed academic medical center with 65,000 patient discharges estimated annually. Prior to SCLBK and CHG bathing interventions, there were 80 CLABSIs and 39 CAUTIs annually. The daily number of patients used in the simulations was 850 patients. ${ }^{3}$ The probability of a patient developing a CAUTI was 0.1257 per 1,000 patient days and 0.2579 per 1,000 patient days for a CLABSI. Simulation results were calculated at steady state. Parameter values are listed in Table 1.

One $\mathrm{CHG}$ bath costs $\$ 8.47$. Patients who do not receive a $\mathrm{CHG}$ bath on a given day are assumed to receive a bath with non-CHG 
Table 1. Parameters Used in the Simulations

\begin{tabular}{|c|c|c|c|}
\hline Parameter & Parameter Symbol & Parameter Value & Source \\
\hline Discharge rate for patients with central lines & $\delta_{\mathrm{CL}}$ & $0.0556(18 \mathrm{~d})$ & Dube et $\mathrm{al}^{6}$ \\
\hline Discharge rate for patients with CAUTI & $\delta_{\text {CAUTI }}$ & $0.0556(18 \mathrm{~d})$ & Al-Hazmi et $\mathrm{al}^{7}$ \\
\hline Discharge rate for patients with central lines and catheters & $\delta_{\text {Both }}$ & $0.0556(18 \mathrm{~d})$ & Dube et $\mathrm{al}^{6}$ \\
\hline Discharge rate for patients with CLABSI & $\delta_{\text {CLABSI }}$ & $0.0417(24 \mathrm{~d})$ & Dube et $\mathrm{al}^{6}$ \\
\hline Discharge rate for patients with catheters & $\delta_{\text {CATH }}$ & 0.1 & Al-Hazmi et $\mathrm{al}^{7}$ \\
\hline Discharge rate for all other patients in the hospital & $\delta_{\text {Other }}$ & 0.2 & Baek et $\mathrm{al}^{10}$ \\
\hline Probability of getting a central line & $\rho_{\mathrm{CL}}$ & 0.01232 & Chopra et $\mathrm{al}^{8}$ \\
\hline Probability of getting a catheter & $\rho_{\text {CATH }}$ & 0.01270 & Carrouget et al $^{9}$ \\
\hline Probability of getting both a central line and a catheter & $\rho_{\text {Both }}$ & 0.0001565 & $\begin{array}{l}\text { Chopra et } \mathrm{al}^{8}, \\
{\text { Carrouget et } \mathrm{al}^{9}}^{9}\end{array}$ \\
\hline Actual infection rate for those who have receieved a central line or both devices in $i$ days & $r_{i}$ & $\left(\frac{1-(3-\mathrm{i}) \cdot \eta \cdot \kappa}{3}\right) r$ & Calculated \\
\hline Actual infection rate for those who have received a catheter $k$ days ago & $r_{k}$ & $\left(\frac{1-(3-i) \cdot \kappa}{3}\right) r$ & Calculated \\
\hline Reduction of incidence of CAUTI and CLABSI due to CHG bathing & $\eta$ & $0.12,0.6$ & Calculated \\
\hline Reduction of CLABSI due to the standardized kit & $\kappa$ & $0.001-0.20$ & Calculated \\
\hline Infection rate & $r$ & $0.00157-0.0007$ & Calculated \\
\hline
\end{tabular}

wipes that cost $\$ 2.47$ per bath. The noncentralized central-line bundle costs $\$ 0.04$ more due to the compilation of necessary supplies needed to insert a central line compared to the SCLBK. On average, a CAUTI infection costs $\$ 13,793^{4}$ and a CLABSI infection costs $\$ 70,696 .^{5}$ The total cost calculation included costs related to CHG bathing materials for CAUTI, the SCLBK for CLABSI, and the costs associated with HAIs.

\section{Results}

Implementation of CHG bathing and SCLBK, and the associated costs, were simulated to be initiated in increments of 6-month delays and were compared no implementation over 5 years. Overall, as the delay in implementation for the infection intervention programs increased, the number of HAIs increased, and the associated savings in healthcare costs by implementation decreased.

Every 6-month delay in improvement of CHG bathing compliance resulted in $\sim 11$ preventable CAUTIs and an additional cost of $\$ 11,000$. Every 6-month delay in implementing the SCLBK resulted in $\sim 10$ CLABSIs and an additional $\$ 715,000$ in costs. Overall during the 5-year period, 102 CLABSIs and 105 CAUTIs could have been prevented, with a savings of $\sim \$ 7.2$ million through CLABSI prevention and $\$ 115,000$ through CAUTI prevention.

\section{Discussion}

Delaying implementation of infection prevention initiatives leads to increased HAIs and total associated healthcare costs. If there are better intervention strategies that are more expensive, they may end up saving money in the end. When the SCLBK and CHG bathing are immediately implemented, healthcare systems can prevent 200 HAIs per year. Each monthly delay leads to decreases in total associated healthcare savings. Lower overall savings for CAUTIs was due to the $\$ 6.00$ difference with the implementation of
CHG compared to a $\$ 0.04$ difference for SCLBK. Also, the healthcare costs dealing with a CAUTI were lower than for CLABSI.

The role of active resistors and organizational constipators in implementing CHG bathing and the SCLBK has a dramatic impact on hospital costs and patient outcomes. Our model was limited by assumptions, such as not including educational and monitoring costs, but it allowed for predictions and quantitative analysis of immediate or delay in implementation of CHG bathing and SCLBK and their effects.

Financial support. No financial support was provided relevant to this article.

Conflicts of interest. All authors report no conflicts of interest relevant to this article.

\section{References}

1. Bearman G, Stevens MP. Pushing beyond resistors and constipators: implementation considerations for infection prevention best practices. Curr Infect Dis Rep 2014;16:388.

2. McMullan C, Propper G, Schuhmacher C, et al. A multidisciplinary approach to reduce central line-associated bloodstream infections. Joint Comm J Qual Patient Saf 2013;39.2:61-AP2.

3. Reagan, KA, Chan, DM, Vanhoozer, G, et al. You get back what you give: decreased hospital infections with improvement in CHG bathing, a mathematical modeling and cost analysis. Am J Infect Control 47:pii:S01966553(19)30683-2.

4. Estimating the additional hospital inpatient cost and mortality associated with selected hospital-acquired conditions. Agency for Healthcare Research and Quality website. https://www.ahrq.gov/hai/pfp/haccost2017. html. Published 2017. Accessed January 28, 2021.

5. Eliminating CLABSI, a national patient safety imperative. Agency for Healthcare Research and Quality website. https://psnet.ahrq.gov/issue/ eliminating-clabsi-national-patient-safety-imperative. Published 2010. Accessed January 28, 2021.

6. Dube WC, Jacob JT, Zheng Z, et al. Comparison of rates of central lineassociated bloodstream infections in patients with 1 vs 2 central venous catheters. JAMA Network Open 2020;3(3):e200396. 
7. Al-Hazmi H. Role of duration of catheterization and length of hospital stay on the rate of catheter-related hospital-acquired urinary tract infections. Res Rep Urol 2015;7:41-77.

8. Chopra V, Govidan S, Kuhn L, et al. Do clinicians know which of their patients have central venous catheters? A multicenter observational study. Ann Intern Med 2014;161:562-567.
9. Carrouget J, Legeay C, Poirier A, Azzouzi A-R, Zahar J-R, Bigot P. Enquête de prévalence sur le sondage vésical dans un centre hospitalo-universitaire. Progrès en Urologie 2017;27(5):305-311.

10. Baek H, Munsu C, Seok K, et al. Analysis of length of hospital stay using electronic health records: a statistical and data mining approach. PloS One 2018;13(4);e0195901.

\title{
Intrapatient transfer of an uncommon carbapenemase in Nebraska
}

\author{
Alyssa K. W. Maclean BS ${ }^{1,2}$ (1), Stacey Morrow MS, MT(ASCP) $)^{1,2}$, Anum Abbas MD ${ }^{3}$ (1), Renuga Vivekanandan $\mathrm{MD}^{3}$ (1), \\ Stephen Cavalieri $\mathrm{PhD}^{1,2,4,5}$ and Nancy D. Hanson $\mathrm{PhD}^{1,2}$ (1) \\ ${ }^{1}$ Department of Medical Microbiology and Immunology, Creighton University School of Medicine, Omaha, Nebraska, ${ }^{2}$ Center for Research in Anti-Infectives and \\ Biotechnology, Creighton University School of Medicine, Omaha, Nebraska, ${ }^{3}$ Division of Infectious Diseases, Creighton University School of Medicine, Omaha, \\ Nebraska, ${ }^{4}$ Department of Pathology, Creighton University School of Medicine, Omaha, Nebraska and ${ }^{5} \mathrm{CHI}$ Health Microbiology Laboratory, Omaha, Nebraska
}

To the Editor-Carbapenem-resistant Enterobacterales are a major medical concern, especially during the coronavirus disease 2019 (COVID-19) pandemic because bacterial superinfections in severe acute respiratory coronavirus virus 2 (SARS-CoV-2)infected patients have led to poor outcomes. ${ }^{1}$ Enterobacterales can emerge resistant to carbapenems through multiple mechanisms including the acquisition of carbapenemase genes on mobile genetic elements such as plasmids. These mobile genetic elements are a major concern due to the potential spread of carbapenem resistance and other resistance elements between multiple bacterial species. ${ }^{2,3}$ Carbapenemase-producing Enterobacterales (CRE) are found worldwide and are widespread in the United States, including in the state of Nebraska. ${ }^{4}$ Although KPC is the most commonly identified carbapenemase in the United States, New Delhi metallo$\beta$-lactamase (NDM) carbapenemases have been reported since $2010^{5}$ and have infected patients with and without history of international travel. ${ }^{3}$ In Nebraska, routine screening for carbapenem resistance has been recommended since 2017. Phenotypic or genotypic confirmation of carbapenemase production is performed by the Nebraska Public Health Lab. ${ }^{6}$ We describe the first documented case of infection with an NDM-7-producing Enterobacterales in the state of Nebraska. Furthermore, this case indicates the potential for plasmid spread to multiple species within a single patient.

Bacterial identification and antimicrobial susceptibility testing were performed using MicroScan Walkaway (Beckman Coulter, Brea, CA). Phenotypic determination of carbapenemase production for CRE was performed using the modified carbapenem inactivation method (mCIM) as described by the Clinical and Laboratory Standards Institute (CLSI). ${ }^{7}$ According to the Nebraska Department of Health and Human Services protocol, carbapenem-resistant isolates were sent to the Nebraska Public Health Laboratory for genotypic determination of carbapenemase production using Xpert Carba-R (Cepheid, Sunnyvale, CA). Following the initial identification and positive $\mathrm{mCIM}$ test, the isolate was sent to our laboratory for further evaluation. Confirmation of the presence of the NDM gene as well as the absence of other

Author for correspondence: Nancy D. Hanson, E-mail: ndhanson@creighton.edu

Cite this article: Maclean AKW, et al. (2022). Intrapatient transfer of an uncommon carbapenemase in Nebraska. Infection Control \& Hospital Epidemiology, 43: 535-536, https://doi.org/10.1017/ice.2021.33 carbapenemase genes was determined using the Streck ARM-D $\beta$-lactamase identification kit according to manufacturer's instructions. The NDM allele was further identified by Sanger sequencing. Plasmid carriage of NDM-7 was confirmed by Southern blotting using NDM-specific probes. ${ }^{8}$

A middle-aged African-American male presented in the emergency room with a left-foot ulcer associated with poorly controlled diabetes melitus type 2 (Fig. 1A). The patient had a long-standing issue with ulcers in his feet and had been followed by a podiatrist for several years. Patient history was significant for previous foot ulcers positive for methicillin-susceptible Staphylococcus aureus and recent travel to the Middle East for work. The diabetic foot ulcer was initially treated in the Middle East with surgical debridement and amputation of the second toe. The patient was prescribed amoxicillin-clavulanate and discharged. While traveling in Nebraska, the patient presented in the emergency room for a wound check. Upon presentation, the left foot had several deep ulcers in stage III and IV that appeared to have good granulation tissue, and no tenderness or purulence was noted. Laboratory tests revealed a normal white blood cell count of $11.810^{3} / \mathrm{mm}^{3}$; elevated creatinine at $2.61 \mathrm{mg} / \mathrm{dL}$ (baseline $2.0 \mathrm{mg} / \mathrm{dL}$ ); C-reactive protein (CRP) of $137 \mathrm{mg} / \mathrm{L}$; erythrocyte sedimentation rate $(E S R)>120 \mathrm{~mm} /$ hour. Cultures of the foot were obtained, and the patient was discharged with topical bacitracin. Upon consultation with the infectious disease physician several days later, further cultures were obtained, and the patient was started on oral levofloxacin.

Initial cultures were positive for carbapenem-resistant Enterobacter cloacae (Figure 1B). Follow-up cultures were positive for carbapenem-resistant Klebsiella pneumoniae and methicillinsusceptible Staphylococcus aureus (susceptibility not shown). For both Enterobacterales isolates, the mCIM test was positive. Realtime polymerase chain reaction (PCR) using the Streck ARM-D $\beta$-lactamase identification kit was positive for NDM in both the E. cloacae isolate (Entb 348) and the K. pneumoniae isolate (Kleb 407). Sanger sequencing of the NDM gene in both isolates identified the NDM-7 carbapenemase gene. Southern blots were performed to determine the location of the NDM-7 gene. Both strains carried a plasmid of the same size encoding NDM-7, suggesting likely conjugative transfer (data not shown). 